\title{
Die deutsch-brasilianischen Beziehungen
}

Peter Birle

\section{Einleitung}

Deutschland und Brasilien sind auf vielfältige Art und Weise miteinander verbunden. Die bilateralen Beziehungen weisen nicht nur eine große historische Tiefe auf, sie beziehen sich auch auf zahlreiche Bereiche - von Politik und Wirtschaft bis hin zu Wissenschaft und Kultur - und zeichnen sich gerade in den vergangenen Jahren durch eine große Dynamik aus. Eine Stärke der Beziehungen liegt darin, dass sie sich nicht nur auf politischdiplomatischer Ebene und zwischen wirtschaftlichen Akteuren abspielen, sondern dass es auch viel wechselseitiges Interesse an der Kultur des Partnerlandes sowie zahlreiche zivilgesellschaftliche Initiativen und Gruppen in beiden Ländern gibt, die im Austausch miteinander stehen. Durch eine Reihe von offiziellen Initiativen wird seit einigen Jahren versucht, die Beziehungen noch weiter auszuweiten und zu vertiefen, die Sichtbarkeit der Zusammenarbeit zu erhöhen und Anstöße für neue Kooperationen zu geben. Im Jahr 2010/2011 fand das "Deutsch-Brasilianische Jahr der Wissenschaft, Technologie und Innovation" statt. Unter dem Motto "Wo Ideen sich verbinden" läuft seit Mai 2013 das Jahr "Deutschland + Brasilien 2013-2014". Im Herbst 2013 ist Brasilien Gastland der Frankfurter Buchmesse, Deutschland präsentiert sich seinerseits auf der Buchmesse von Rio de Janeiro.

Der folgende Beitrag geht zunächst kurz auf einige historische Aspekte der bilateralen Beziehungen ein und analysiert dann die Zusammenarbeit in den Bereichen Politik, Wirtschaft, Wissenschaft und Forschung, Kultur und Entwicklungszusammenarbeit. Ein Leitmotiv ist dabei die Frage nach den Ursachen dafür, dass Deutschland und Brasilien sich trotz großer Unterschiede und divergierender außenpolitischer Prioritäten als 'strategische Partner' betrachten und in vielen Bereichen eine enge Zusammenarbeit suchen. 


\section{Ein kurzer Blick zurück}

Bereits seit dem 16. Jahrhundert und damit lange vor der staatlichen Unabhängigkeit Brasiliens waren deutsche Wissenschaftler, Geistliche und Soldaten in dem unter portugiesischer Kolonialherrschaft stehenden Brasilien aktiv. Nach der Unabhängigkeit im Jahr 1822 förderte das Kaiserreich gezielt die Anwerbung deutscher Söldner für das neu zu gründende Heer und die Einwanderung deutscher Siedler. Vor allem im Süden des Landes gelegene Regionen wie Rio Grande do Sul und Santa Catarina sollten durch die Ansiedlung von Migranten gegen mögliche Angriffe von außen geschützt werden. In mehreren Wellen wanderten im Laufe des 19. und 20. Jahrhunderts Hunderttausende von Deutschen nach Brasilien aus. Sie trugen zur landwirtschaftlichen Erschließung bei und prägten als Kleinbauern den ländlichen Raum in nicht unerheblichem Maße. Die Nachfahren der Migranten hinterließen in der brasilianischen Wirtschaft und Kultur deutliche Spuren und trugen mit dazu bei, dass die Beziehungen zwischen beiden Ländern heute über ein solides Fundament individueller und zivilgesellschaftlicher Verbindungen verfügen. Auch wenn die Kenntnis der deutschen Sprache heutzutage rückläufig ist, so weisen doch viele Brasilianer deutsche Wurzeln auf und vor allem im Süden und Südosten des Landes ist der kulturelle Einfluss der Einwanderung nach wie vor spürbar.

Brasilien ist schon früh ein Land gewesen, für das sich deutsche Wissenschaftler und Künstler interessierten. Naturforscher wie Carl Friedrich Philipp von Martius, Karl von den Steinen und Theodor Koch-Grünberg trugen dazu bei, dass die deutsche Brasilienforschung im 19. und frühen 20. Jahrhundert einen international hervorragenden Ruf genoss.

Auch die wirtschaftlichen Beziehungen setzten schon bald nach der Unabhängigkeit Brasiliens ein und waren im 19. Jahrhundert umfassender als die zwischen Deutschland und den USA. In den 20er Jahren des 19. Jahrhunderts avancierte Deutschland zum wichtigsten Abnehmer von brasilianischem Zucker und Kaffee. Auch andere Rohstoffe wie Tabak, Baumwolle und Leder wurden importiert. Schon früh zeigte sich im Hinblick auf die Wirtschaftsbeziehungen ein Aspekt, der bis zum heutigen Tag ein Charakteristikum der brasilianischen Außen- und Außenwirtschaftspolitik ist: Man war und ist stets darum bemüht, einseitige politische und wirtschaftliche Abhängigkeiten zu vermeiden oder zumindest gegen sie anzukämpfen und hat es in diesem Zusammenhang oft verstanden, sich Rivalitäten zwischen anderen Ländern zu Nutze zu machen. In diesem 
Sinne waren die Wirtschaftsbeziehungen mit Deutschland ein wichtiges Gegengewicht, um die seit Ende des 19. Jahrhunderts zunehmende wirtschaftliche Abhängigkeit von den USA nicht zu groß werden zu lassen.

Die beiden Weltkriege führten jeweils zu einer zeitweisen Unterbrechung der bilateralen Handels- und Wirtschaftsbeziehungen. Gleichwohl erfuhren gerade die Wirtschaftsbeziehungen zwischen den beiden Ländern schon wenige Jahre nach dem Ende des Zweiten Weltkrieges erneut einen enormen Aufschwung. Ab 1953 investierten deutsche Unternehmen wie Krupp, Mercedes Benz, Volkswagen und Oetker massiv in Brasilien und gründeten dort Niederlassungen. Brasilien war zu dieser Zeit nicht das einzige lateinamerikanische Land, an dem die deutsche Wirtschaft ein großes Interesse zeigte. Insgesamt flossen in den 1950er Jahren mehr deutsche Direktinvestitionen nach Lateinamerika als in europäische Länder. Brasilien war dabei mit ungefähr einem Viertel der Investitionen in der Region das bevorzugte Zielland. Für Brasilien wiederum bedeuteten diese mit dem Wiederaufstieg Deutschlands als Handelsmacht verbundenen Investitionen die Möglichkeit, die eigene Industrialisierung trotz Devisenknappheit voranzutreiben und so die entwicklungsstrategischen Zielsetzungen des Landes umzusetzen. Zudem veranlassten die deutschen Investitionen auch die US-amerikanische Konkurrenz, aus Sorge um den brasilianischen Markt dort mehr zu investieren, als dies sonst der Fall gewesen sein dürfte.

Die Machtübernahme durch die Militärs ab 1964 beeinträchtigte die bilateralen Beziehungen kaum, vielmehr wurden einige der bis heute gültigen vertraglichen Grundlagen, beispielsweise das Kulturabkommen (1969), während der Zeit der Diktatur abgeschlossen. Dies gilt auch für das "Abkommen auf dem Gebiet der friedlichen Nutzung der Kernenergie" von 1975. Anders als die USA oder Frankreich war die Bundesrepublik Deutschland damals dazu bereit, sich auf einen Vertrag einzulassen, der die Implementierung des gesamten nuklearen Kreislaufes in Brasilien vorsah. Die USA hatten einen derartigen Technologietransfer aus sicherheitspolitischen Überlegungen abgelehnt. Die Bundesrepublik Deutschland, die bereits seit den 1950er Jahren mit Brasilien im Bereich der Kernenergie kooperierte, erhoffte sich von dem Vertrag Impulse für den Export von Nukleartechnologie und nahm dafür in Kauf, sich erstmals seit dem Ende des Zweiten Weltkrieges wegen ihrer Lateinamerikapolitik auf einen Konflikt mit den USA einzulassen. Brasilien ging es demgegenüber vor allem darum, die eigene nukleare Autonomie voranzutreiben und sich so von einer technologischen Bevormundung durch die USA zu emanzipieren. Die Beherrschung 
des atomaren Kreislaufes galt zudem als unabdingbare Voraussetzung für den von den Militärs angestrebten Großmachtstatus. In diesem Zusammenhang spielte nicht zuletzt die Ausbildung von brasilianischen Atomspezialisten in deutschen Forschungseinrichtungen eine wichtige Rolle. Auch wenn die vertraglich vereinbarte Zusammenarbeit im Nuklearbereich aus unterschiedlichen Gründen weit hinter den ursprünglichen Plänen zurückblieb, ziehen beide Seiten bis heute letztlich eine positive Bilanz aus dem Projekt, nicht zuletzt deshalb, weil sich daraus viele Impulse für die wirtschaftliche und technologische Kooperation in anderen Bereichen ergaben.

\section{Die politischen Beziehungen}

Weder für Deutschland noch für Brasilien nehmen die wechselseitigen bilateralen Beziehungen einen vorderen Rang in den jeweiligen außenpolitischen Prioritätensetzungen ein. Die deutsche Außenpolitik gilt zunächst Europa und der transatlantischen Partnerschaft mit den USA. Richtschnur für das außen- und sicherheitspolitische Handeln ist die Einbindung in die Vereinten Nationen (VN), die Organisation für Sicherheit und Zusammenarbeit in Europa (OSZE), die North Atlantic Treaty Organization (NATO) und die Europäische Union (EU). Darüber hinaus richten sich die Blicke auf die EU-Nachbarschaftspolitik gegenüber Osteuropa und den Mittelmeeranrainern, auf die "Transformationspartnerschaften" mit den Staaten Nordafrikas, auf den Nahostkonflikt sowie auf Krisenländer wie Afghanistan, Mali, Syrien, und Iran. Demgegenüber gilt die gegenwärtige brasilianische Außenpolitik in erster Linie Lateinamerika und Afrika. Entwicklung, Frieden, die Reduzierung der Kluft zwischen reichen und armen Ländern, Gleichheit zwischen den Völkern und eine effektive Demokratisierung des internationalen Systems, so lauten ihre wichtigsten Ziele. Neben der regionalen Zusammenarbeit in Lateinamerika, wozu neben dem Gemeinsamen Markt des Südens (MERCOSUL) vor allem das Engagement im Rahmen der Union Südamerikanischer Nationen (UNASUL) sowie der Gemeinschaft der Lateinamerikanischen und Karibischen Staaten (CELAC) gehört, ist Brasilien in multilateralen Süd-Süd-Foren wie der BRICS-Gruppe (Brasilien, Russland, Indien, China und Südafrika) und dem IBSA-Forum (Indien, Brasilien, Südafrika) aktiv.

Gleichwohl sind die deutsch-brasilianischen Beziehungen für beide Länder auch in politisch-diplomatischer Hinsicht alles andere als marginal. 
Deutschland ist neben Frankreich Brasiliens wichtigster Partner in Europa, umgekehrt ist Brasilien neben Mexiko der wichtigste Ansprechpartner Deutschlands in Lateinamerika. Beide Länder interessieren sich, wenn auch aus zum Teil unterschiedlichen Motiven, für eine Vertiefung der bilateralen Beziehungen, das zeigt auch die rege Besuchsdiplomatie der vergangenen Jahre. Die Regierungen von Bundeskanzlerin Angela Merkel und Präsident Luiz Inácio Lula da Silva unterzeichneten im Mai 2008 in der brasilianischen Hauptstadt Brasilia einen "Aktionsplan der deutsch-brasilianischen strategischen Partnerschaft". Darin bekräftigen beide Länder die "Entschlossenheit, ihre strategische Partnerschaft durch den Ausbau der bilateralen Beziehungen auf allen Ebenen und in verschiedenen Bereichen weiter zu vertiefen" (Aktionsplan 2008: 1). Der Plan sieht neben einem regelmäßigen politischen Dialog, in den auch Themen der internationalen Agenda einbezogen werden sollen, folgende Kooperationsbereiche vor:

- VN-Reform/Kooperation in multilateralen Foren

- Sicherheitspolitik, Abrüstung, Nichtverbreitung von Atomwaffen

- Menschenrechte

- Bekämpfung der organisierten Kriminalität/Terrorismus/Rechtshilfe

- Multilaterale Handelsbeziehungen

- Stabiles internationales Finanzsystem

- Engere Beziehungen zwischen der EU und Lateinamerika

- Bilaterale Wirtschaftsbeziehungen

- Deutsch-brasilianische Energiezusammenarbeit

- Klimawandel

- Nachhaltige Entwicklung und Umwelt/Bilaterale Zusammenarbeit

- Bilaterale Kulturbeziehungen/Bildungszusammenarbeit

- Forschung für Nachhaltigkeit/Hochtechnologie

- Dialog der Gesellschaften

Brasilien gehört in den Augen der Bundesregierung zu einer Gruppe von Ländern, die seit 2012 als "neue Gestaltungsmächte" bezeichnet werden:

Die Ordnung der internationalen Staatengemeinschaft wird zunehmend multipolar: Einflussreiche Länder, die lange als Entwicklungs- oder Schwellenländer bezeichnet wurden, gestalten internationale Politik in einer interdependenten Welt. Sie sind wirtschaftliche Lokomotiven, sie beeinflussen 
maßgeblich die Zusammenarbeit in ihren Regionen, sie wirken auch in anderen Weltregionen und sie spielen in internationalen Entscheidungsprozessen eine zunehmend wichtige Rolle. Selbstbewusst finden sie ihren Platz in den internationalen Beziehungen und übernehmen zunehmend Verantwortung für globale Fragen. Wir sehen in ihnen mehr als Schwellenländer: Sie sind 'neue Gestaltungsmächte'. (Auswärtiges Amt 2012: 5)

Wenn Deutschland sich Brasilien als Partner für die Gestaltung globaler Prozesse anbietet, dann steckt dahinter nicht nur Anerkennung dafür, dass Brasilien sich in jüngerer Zeit auf der Grundlage einer dialogorientierten und kooperativen Außenpolitik sowohl in Lateinamerika als auch auf globaler Ebene konstruktiv engagiert, Impulse für notwendige Reformen gegeben und zunehmende politische Verantwortung übernommen hat. Wie das Zitat zeigt, steht das Zugehen auf Länder wie Brasilien auch im Zusammenhang mit den von Seiten der Bundesregierung wahrgenommenen Veränderungen des internationalen Systems insgesamt. Eine Rolle dürfte dabei auch das Bewusstsein spielen, dass sich die relative Bedeutung Deutschlands auf globaler Ebene aufgrund globaler wirtschaftlicher und demographischer Trends in den kommenden Jahrzehnten eher verringern, das Gewicht Brasiliens dagegen weiter vergrößern wird. Eine vernetzte Zusammenarbeit mit Brasilien ist daher auch ein Stück Zukunftsvorsorge, d. h. es geht um die Festigung von soliden bilateralen Verbindungen, um nachhaltig für die eigenen Positionen zu werben und auch zukünftig globale Strukturen mitgestalten zu können. Dies darf allerdings nicht darüber hinwegtäuschen, dass im Hinblick auf die im Rahmen des "Aktionsplans der deutsch-brasilianischen strategischen Partnerschaft" genannten Kooperationsbereiche nur zum Teil übereinstimmende Interessen bestehen. Insofern scheint es stark übertrieben, wenn das Auswärtige Amt auf seiner Website pauschal davon spricht, dass die bilateralen Beziehungen "auf gemeinsamen Werten und übereinstimmenden Auffassungen zur globalen Ordnung" beruhen. Für einen Teil des Wertekanons (Demokratie, Menschenrechte, friedliche Konfliktlösung, etc.) und Teilbereiche der internationalen Ordnung trifft dies in der Tat zu, etwa für die Reform der VN, im Hinblick auf die beide Länder ihre gegenseitige Unterstützung bei der Kandidatur für einen ständigen Sitz in einem erweiterten Sicherheitsrat nachdrücklich bekräftigen. In anderen Bereichen besteht zwar Konsens über die Notwendigkeit von Reformen, aber über deren konkrete Ausgestaltung gehen die Ansichten auseinander. Im Hinblick auf den von Brasilien geforderten besseren Zugang zum europäischen Binnenmarkt vor allem im Bereich der Landwirtschaft ist Deutschland zwar grundsätzlich reformbereit, wird deswegen aber kaum 
einen Konflikt mit denjenigen EU-Partnerländern riskieren, die entsprechende Reformen ablehnen. Die Interessendivergenzen zwischen Brasilien und Deutschland hinsichtlich der Gestaltung globaler Strukturen und Prozesse hängen in erster Linie damit zusammen, dass Brasilien den Status Quo (beispielsweise in puncto Welthandelsordnung oder Stimmenanteile in Weltbank und Internationalem Währungsfonds) als ungerecht empfindet und auf eine stärkere Beteiligung der Länder des Südens drängt, während Deutschland zwar ein deutlicher Verfechter des Multilateralismus ist, aber nicht für eine grundlegende Revision des Status Quo eintritt.

Unterschiedliche Vorstellungen gibt es auch im Hinblick auf die Rolle der nationalen Souveränität. Die Bundesrepublik Deutschland hat sich nach dem Zweiten Weltkrieg nicht nur an die Erfahrung gewöhnt, mit einer durch die Siegermächte in Teilen beschränkten nationalen Souveränität zu leben; sie hat auch im Rahmen des europäischen Integrationsprozesses nationale Souveränitätsrechte auf europäische Instanzen übertragen und ist davon überzeugt, dass dieses Pooling von Souveränität im eigenen Interesse ist, da dadurch schlagkräftige supranationale Instanzen entstehen. Man sollte die in Europa vorhandene Bereitschaft zur Abgabe von Souveränität nicht idealisieren, denn die großen Schwierigkeiten im Hinblick auf die Etablierung einer gemeinsamen europäischen Außenund Sicherheitspolitik zeigen sehr deutlich, dass auch hier die Bereitschaft zur Abgabe von Souveränität klare Grenzen kennt. Sie ist aber zumindest in einigen Politikbereichen vorhanden.

Die lateinamerikanischen Länder haben demgegenüber völlig andere historische Erfahrungen gemacht, die auch ihre heutigen außenpolitischen Selbstverständnisse prägen: Nicht nur die jahrhundertelange europäische Kolonialherrschaft, sondern auch die Rückzugskämpfe der ehemaligen Kolonialmächte während des 19. Jahrhunderts und vor allem die den größten Teil des 20. Jahrhunderts andauernde hegemoniale Rolle der USA in der westlichen Hemisphäre haben dazu geführt, dass das Bewusstsein einer von außen verweigerten Souveränität sich tief in die nationalen Selbstverständnisse eingeprägt hat. Die im Laufe des vergangenen Jahrzehnts zu beobachtenden Bemühungen um die Etablierung genuin lateinamerikanischer Regionalorganisationen wie UNASUL und CELAC sind nicht zuletzt Ausdruck des Versuchs, sich von den USA zu emanzipieren. Auch wenn die brasilianische Souveränität sicherlich nie derart durch die USA oder andere externe Mächte bedroht war wie beispielsweise die der zentralamerikanischen Staaten, so ist doch auch und gerade für Brasilien der Respekt vor 
der nationalen Souveränität ein zentrales Element seines außenpolitischen Selbstverständnisses. Nicht nur im Hinblick auf die regionalen Kooperationsprozesse in Lateinamerika lehnt das Land jede Abgabe von nationaler Souveränität an übergeordnete Instanzen (etwa im Rahmen von MERCOSUL oder UNASUR) ab; ganz besonders empfindlich reagiert man, wenn beispielsweise im Hinblick auf den Schutz des tropischen Regenwaldes im Amazonas oder in puncto globale Sozial- und Umweltstandards Vorschläge unterbreitet werden, die als "Einmischung in die inneren Angelegenheiten" empfunden werden. Diese divergierenden Souveränitätsverständnisse machen bi- und multilaterale Aushandlungsprozesse mit Brasilien einigermaßen kompliziert. Gleichwohl sind sie kein Hindernis für eine Partnerschaft, die sowohl von Deutschland als auch von Brasilien als wichtig und zukunftsfähig betrachtet wird. Dabei spielt aus brasilianischer Perspektive auch die Tatsache eine Rolle, dass Deutschland sich im Zuge der internationalen Finanzkrise und der Euro-Krise als ein relativ krisenfestes Land erwiesen hat, dessen Bedeutung innerhalb der EU noch zugenommen hat.

\section{Übersicht: Verträge und politische Dokumente zwischen Deutschland und Brasilien (Auswahl)}

\begin{tabular}{|l|}
\hline Abkommen über Soziale Sicherheit (03.12.2009) \\
\hline $\begin{array}{l}\text { Aktionsplan der deutsch-brasilianischen Strategischen Partnerschaft } \\
(14.05 .2008)\end{array}$ \\
\hline $\begin{array}{l}\text { Rahmenabkommen über Zusammenarbeit in der wissenschaftlichen Forschung } \\
\text { und technologischen Entwicklung (20.03.1996) }\end{array}$ \\
\hline Rahmenabkommen über Technische Zusammenarbeit (17.09.1996) \\
\hline $\begin{array}{l}\text { Vereinbarung über den Austausch technischer Informationen und Zusam- } \\
\text { menarbeit auf dem Gebiet der Sicherheit kerntechnischer Einrichtungen } \\
(10.03 .1978)\end{array}$ \\
\hline $\begin{array}{l}\text { Abkommen auf dem Gebiet der friedlichen Nutzung der Kernenergie } \\
(27.06 .1975)\end{array}$ \\
\hline Kulturabkommen (09.06.1969) \\
\hline Basisabkommen über die Technische Zusammenarbeit (30.11.1963) \\
\hline Abkommen über den planmäßigen Luftverkehr (29.08.1957) \\
\hline Briefwechsel über deutsche Investitionen (04.09.1953) \\
\hline $\begin{array}{l}\text { Abkommen über die Wiederherstellung der durch den Zweiten Weltkrieg } \\
\text { betroffenen gewerblichen Schutzrechte und Urheberrechte (04.09.1953) }\end{array}$ \\
\hline
\end{tabular}




\section{Die wirtschaftlichen Beziehungen}

Brasilien ist für die deutsche Wirtschaft ein wichtiger Exportmarkt und Investitionsstandort. Zwar ist das relative Gewicht des Landes in der deutschen Außenhandels- und Investitionsstatistik heute geringer als von Mitte der 1950er bis in die 1970er Jahre, aber nach einem Einbruch in den 1980er Jahren gilt Brasilien seit der erfolgreichen Stabilisierungspolitik unter Präsident Fernando Henrique Cardoso (1995-2002) und spätestens mit den wirtschaftlichen Erfolgen des vergangen Jahrzehnts als ein Markt, auf dem sich gute Geschäfte machen lassen. Der bilaterale Außenhandel Brasilien-Deutschland erreicht gegenwärtig eine Größenordnung von ca. 22 Mrd. $€$ pro Jahr. 2012 hat Deutschland Waren im Wert von 11,7 Mrd. Euro nach Brasilien exportiert. Die Importe aus Brasilien beliefen sich im gleichen Zeitraum auf 10,6 Mrd. Euro. Damit lag Brasilien bei den deutschen Einfuhren auf Rang 21 und bei den Ausfuhren auf Rang 20. Exportiert wurden vor allem Maschinen $(27,8 \%)$, chemische Erzeugnisse $(26,7 \%)$, Kraftfahrzeuge und -teile $(11,0 \%)$ sowie Elektrotechnik (6,7\%), die Importe setzten sich in erster Linie aus Rohstoffen (37,6\%), Nahrungsmitteln (25,7\%), Maschinen (7,0\%), chemischen Erzeugnissen $(6,6 \%)$ sowie Eisen und Stahl (5,3\%) zusammen. Mit 6,4\% Importanteil lag Deutschland 2012 hinter den USA (15,3\%), China (14,6\%) und Argentinien (7,4\%) an vierter Stelle der Lieferländer. Bei den Abnehmerländern rangierte Deutschland mit einem Anteil von 3,0 \% an den brasilianischen Exporten nach China (17,0 \%), den USA (11,1\%), Argentinien $(7,4 \%)$, den Niederlanden $(6,2 \%)$ und Japan $(3,3 \%)$ an sechster Stelle. Der Bestand an deutschen Direktinvestitionen in Brasilien betrug 2011 24,222 Mrd. €, wobei der Nettotransfer seit 2010 jeweils negativ war (2010: -949; 2011: -2.011; 2012: -2.029). Der Bestand an brasilianischen Direktinvestitionen in Deutschland ist bislang relativ bescheiden, er betrug 2010162 Mio. €, der Nettotransfer belief sich 2010 auf 51 Mio., 2011 auf 52 Mio. und 2012 auf -10 Mio. $€$ (Germany Trade \& Invest 2013). 
Tabelle 1: Der deutsche Außenhandel mit Brasilien, 2010-2012

\begin{tabular}{|r|c|c|c|c|c|c|}
\hline & 2010 & $\%$ & 2011 & $\%$ & 2012 & $\%$ \\
\hline $\begin{array}{r}\text { Dt. } \\
\text { Einfuhr }\end{array}$ & 9,4 & 30,6 & 11,3 & 20,2 & 10,6 & $-6,2$ \\
\hline $\begin{array}{r}\text { Dt. } \\
\text { Ausfuhr }\end{array}$ & 10,4 & 42,5 & 11,2 & 7,7 & 11,7 & 4,5 \\
\hline Saldo & 1,0 & & $-0,1$ & & 1,1 & \\
\hline
\end{tabular}

Quelle: Germany Trade \& Invest 2013: 4.

Die relative Bedeutung Brasiliens für die deutsche Außenwirtschaft hat sich seit den 1990er Jahren kaum verändert. Dies ist insofern überraschend, als gerade in den letzten Jahren allerorten von einem "BrasilienBoom" die Rede ist. So heißt es in einer an die mittelständische Industrie gerichteten Broschüre aus dem Jahr 2012 unter der Überschrift "Chancen nutzen - Netzwerke ausbauen":

Brasilien ist in aller Munde - sechstgrößte Volkswirtschaft weltweit, kontinentales Schwergewicht, Agrarweltmacht und Rohstoffgigant. Das Land ist längst kein Zukunftsmarkt mehr - es ist vielmehr Ort der Gegenwart und Region voller Chancen, die es zu nutzen gilt. Angesichts des enormen Potenzials stellt sich für viele Unternehmer mittlerweile nicht mehr die Frage, ob investiert werden soll, sondern wie der Markteintritt gelingen kann. (Rödl \& Partner 2012: 9)

Schätzungsweise 1.400 deutsche Unternehmen sind bereits in Brasilien aktiv, ein großer Teil von ihnen hat sich im Großraum São Paulo angesiedelt. In einigen Branchen, etwa in der Automobilindustrie, im Maschinenbau sowie bei Chemie und Pharma nehmen sie Spitzenpositionen ein. Die drei Deutsch-Brasilianischen Industrie- und Handelskammern in São Paulo, Rio de Janeiro und Porto Alegre bieten ihren Mitgliedern ein umfassendes Dienstleistungsangebot, das sich nicht nur an deutsche Unternehmen richtet, die sich in Brasilien niedergelassen haben oder dies planen, sondern auch an brasilianische Unternehmen mit Interesse am deutschen Markt (<www.ahkbrasilien.com.br>). Nicht zuletzt mit Blick auf die bevorstehenden sportlichen Großereignisse FIFA-WM 2014 und Olympische Spiele 2016 hat der Bundesverband der Deutschen Industrie (BDI) zusätzlich zu den bestehenden Strukturen, zu denen auch die seit über 30 Jahren 
jährlich stattfindenden Deutsch-Brasilianischen Wirtschaftstage gehören, ein Brazil Board geschaffen, das das Brasilienengagement der deutsche Industrie bündeln soll und sich "für eine konsequente und langfristige Intensivierung der deutsch-brasilianischen Wirtschaftsbeziehungen, vor allem über strategische Partnerschaften" (BDI 2012: 1) einsetzt. Nicht zuletzt geht es solchen Lobby-Organisationen natürlich auch darum, gegenüber den Regierungen der beiden Länder jene Aspekte zu thematisieren, die als Haupthindernisse für ein noch stärkeres Engagement der deutschen Wirtschaft in Brasilien empfunden werden: die staatliche Bürokratie, das komplizierte Steuersystem, das fehlende Doppelbesteuerungsabkommen, die komplexen Zoll-, Import- und Handelsbestimmungen sowie Probleme in den Bereichen Transport und Infrastruktur.

Aus brasilianischer Perspektive ist das Engagement deutscher Unternehmen in Brasilien vor allem deshalb interessant, weil man sich davon weitere Technologie- und Innovationsschübe erwartet. Deutsche Unternehmen tragen erheblich zur Ausbildung hochqualifizierter brasilianischer Ingenieure und Techniker bei und der von ihnen generierte Mehrwert kommt nicht nur den jeweiligen Unternehmensbilanzen zugute, sondern er bedeutet auch für die brasilianische Volkswirtschaft einen Kompetitivitätszuwachs auf den internationalen Märkten. Dies ist gerade für ein Land wie Brasilien, das sich nicht damit begnügen will, Rohstoffe auszuführen und wissensintensive Produkte zu importieren, ein wertvoller Beitrag zur Verwirklichung einer langfristig orientierten Entwicklungsstrategie (Vargas 2011).

\section{Die Zusammenarbeit in Wissenschaft und Forschung}

Zwischen Deutschland und Brasilien existiert ein gut ausgebautes Kooperationsnetzwerk in den Bereichen Wissenschaft und Forschung. Die wichtigsten institutionellen Akteure auf deutscher Seite sind dabei der Deutsche Akademische Austauschdienst (DAAD), die Deutsche Forschungsgemeinschaft (DFG) sowie das Bundesministerium für Bildung und Forschung (BMBF) und das Auswärtige Amt (AA). Seit Februar 2012 existiert als gemeinsame Vertretung das Deutsche Wissenschafts- und Innovationshaus (DIWH) in São Paulo. Ähnliche Einrichtungen gibt es sonst nur noch in New York, Moskau, Neu-Delhi, Tokio und Kairo, auch dies ist schon eine Aussage über die zunehmende Bedeutung, die Brasilien als Partner im Bereich von Wissenschaft und Forschung eingeräumt 
wird. Durch die gemeinsame Vertretung der deutschen Wissenschaft und Innovation an einem Ort sollen Synergien zwischen den deutschen Organisationen erzeugt werden. Zudem geht es darum, die Sichtbarkeit und Zugänglichkeit der deutschen Forschungs- und Wissenschaftslandschaft für brasilianische Interessenten zu erhöhen ( $<$ dwih.com.br $>$ ).

Als beispielhaft für die quantitative und qualitative Entwicklung der deutsch-brasilianischen Wissenschaftskooperation seit den 1950er Jahren können die Phasen betrachtet werden, die Gabriele Althoff für das Engagement des DAAD in Brasilien identifiziert hat (zum Folgenden siehe Althoff 2010: 693f.). Seit dem Beginn der DAAD-Aktivitäten in Brasilien bis Mitte der 1970er Jahre bestand die Kooperation in erster Linie in einem einseitigen Stipendienangebot von deutscher Seite sowie aus relativ zufälligen Fördermaßnahmen für Deutsche und Brasilianer. Nach der Unterzeichnung eines Abkommens über den Wissenschaftleraustausch mit dem $\mathrm{Na}$ tionalen Rat für Forschung und Technologie (CNPq) im Jahr 1974 erfolgte eine Erhöhung des Austauschvolumens (jährlich bis zu 100 Wissenschaftler sowie 30 bis 40 Kurzzeitdozenten, Lektoren und Langzeitdozenten). Ein entscheidender quantitativer und qualitativer Sprung der Zusammenarbeit ergab sich dann seit Mitte der 1990er Jahre. Auf brasilianischer Seite wurde die Stiftung zur Förderung des Hochschulnachwuchses (CAPES) zum wichtigsten Kooperationspartner des DAAD und gleichzeitig entstanden in der Folgezeit eine Reihe von neuen, gemeinsam finanzierten Programmen, durch die sich die Schwerpunkte der Zusammenarbeit von der Individualförderung auf die projektbezogene Förderung verlagerten. Das wichtigste Programm in diesem Zusammenhang ist PROBRAL, zusätzlich besteht seit 2001 ein Programm zur Zusammenarbeit in der universitären Lehre, UNIBRAL. Seit 2008 gibt es eine Vereinbarung über ein gemeinsames Programm für Doppelabschlüsse und Doppelpromotionen, seit 2009 ein Abkommen über eine "Strategische Partnerschaft in Forschung und Lehre".

Auch die DFG hat ihr Brasilienengagement seit Mitte des vergangenen Jahrzehnts erheblich ausgeweitet. Seit 2006 existiert ein Abkommen mit der Stiftung zur Förderung der Forschung im Bundesstaat São Paulo (FAPESP), auf dessen Basis zahlreiche Forschungskooperationen gefördert werden. Weitere deutsche Wissenschaftseinrichtungen, die sich in Brasilien engagieren, sind beispielsweise die Alexander von Humboldt Stiftung und die Fraunhofer-Gesellschaft. Einige deutsche Bundesländer sind mit eigenen Initiativen hervorgetreten. So wurde vor gut zehn Jahren das Baden-Württembergische Brasilien-Zentrum eingerichtet, um die wis- 
senschaftliche, technische und kulturelle Kooperation zwischen BadenWürttemberg und Rio Grande do Sul zu fördern und auszubauen (<www. uni-tuebingen.de/brasilien-zentrum $>$ ). Auch das Bayerische Hochschulzentrum für Lateinamerika (BAYLAT), eine Serviceeinrichtung zur Förderung der internationalen Vernetzung von bayerischen und lateinamerikanischen Hochschulen und zur Werbung für den Standort Bayern als Zentrum für Technologie und Innovation sowie Wissenschaft und Lehre, weist einen starken Brasilienbezug auf (<www.baylat.org/>). Zwischen deutschen und brasilianischen Hochschulen bestanden laut Angaben der Hochschulrektorenkonferenz Anfang Juli 2013427 Kooperationsabkommen.

Die deutsch-brasilianische Zusammenarbeit in Wissenschaft und Forschung zeichnet sich in den vergangenen Jahren durch eine sehr dynamische Entwicklung aus. Auch die brasilianische Seite ergreift dabei zunehmend die Initiative für eine Vertiefung der Kooperation. Ein Beleg dafür ist das von der Regierung Rousseff ins Leben gerufene Mobilitätsprogramm "Wissenschaft ohne Grenzen", mit dem bis 2014 über 100.000 Studierende und Forscher an führende Universitäten weltweit entsandt werden sollen. Rund 10.000 Stipendiatinnen und Stipendiaten sollen allein nach Deutschland kommen, damit liegt Deutschland hinter den USA und Großbritannien auf Platz drei der Zielländer. Allerdings fällt ein starkes Übergewicht der Bereiche Naturwissenschaft und Technik in der bilateralen Kooperation auf. Hier liegen auch eindeutig die Prioritäten sowohl der beiden Regierungen als auch der Wirtschaft. Demgegenüber fällt die Kooperation im Bereich der Sozial- und Geisteswissenschaften deutlich bescheidener aus. Lehre und Forschung zu Brasilien an deutschen Hochschulen sind in den meisten sozial- und geisteswissenschaftlichen Fächern bei Weitem nicht so umfassend, wie man dies aufgrund der Größe und Bedeutung des südamerikanischen Landes vermuten könnte, auch wenn sich in den vergangenen Jahren ein wachsendes Interesse an Brasilien abzeichnet (Göbel/Birle/Specht 2009). Einrichtungen, die sich in diesem Bereich engagieren, sind beispielsweise das am Lateinamerika-Institut der Freien Universität Berlin 2010 gegründete fachgebietsübergreifende Forschungszentrum Brasilien, das brasilienbezogene Projekte und Forschungsaktivitäten mit kultur- und sozialwissenschaftlicher Ausrichtung bündelt (<www.lai.fu-berlin.de/brasil>), sowie das Martius-Staden-Institut für Wissenschaft, Literatur und deutsch-brasilianischen Kulturaustausch in São Paulo (<www.martiusstaden.org.br>). 


\section{Die Kulturbeziehungen}

Der kulturelle Austausch zwischen Deutschland und Brasilien hat in den vergangenen Jahren einen Aufschwung erlebt, er ist allerdings durch deutliche Asymmetrien gekennzeichnet. Deutschland verfügt über eine auswärtige Kultur- und Bildungspolitik und über sogenannte Kulturmittler, allen voran das Goethe-Institut, deren Aufgabe es ist, weltweit die Kenntnis der deutschen Sprache zu fördern, die internationale kulturelle Zusammenarbeit zu pflegen und durch Information über die deutsche Kultur, Gesellschaft und Politik ein umfassendes Deutschlandbild zu vermitteln. In Brasilien besteht ein relativ enges Netz von Kulturmittlern. Das Goethe-Institut ist in São Paulo, Curitiba, Porto Alegre, Rio de Janeiro und Salvador vertreten, hinzu kommt ein Goethe-Zentrum in Brasilia (<www.goethe.de/brasilien>). Drei deutsche Auslandsschulen, das Colégio Visconde de Porto Seguro und das Colégio Humboldt in São Paulo und die Escola Alemã Corcovado in Rio de Janeiro, führen zur deutschen Hochschulreife, weitere Schulen bieten Deutschunterricht an. Die genannten Organisationen tragen dazu bei, die deutsche Sprache und Literatur, Musik, Kunst, Theater und Film in Brasilien zu verbreiten. ${ }^{1}$

Demgegenüber geschieht die Vermittlung der brasilianischen Kultur in Deutschland sehr viel stärker über den Markt: Schallplattenfirmen und Verlage schicken bekannte Künstler auf Tournee ins Ausland, brasilianische Filme werden auf internationalen Festivals präsentiert. Bis vor etwa zehn Jahren gab es ein Brasilianisches Kulturinstitut in Deutschland (ICBRA), es wurde jedoch geschlossen und seine Aufgaben in die Brasilianische Botschaft in Deutschland zurückverlagert. Dies entspricht der traditionellen Vertretung der brasilianischen Kultur im Ausland durch das Außenministerium. Hinzu kommt, dass Brasilien in puncto Kulturvermittlung andere Prioritäten setzen muss als Deutschland. Ein Land, in dem $87 \%$ der Bevölkerung noch nie im Kino waren, $92 \%$ noch nie in einem Museum und $93 \%$ noch nie auf einer Kunstausstellung (Dantas 2010: 333), muss zunächst die Kulturvermittlung im eigenen Land ankurbeln. Danach liegen die Prioritäten in Lateinamerika sowie in einigen afrikanischen Staaten, vor allem in

1 Siehe dazu die Beiträge in Bader 2010. Sie gehen auf ein 2008 in São Paulo durchgeführtes Symposium zurück, bei dem eine Bestandsaufnahme relevanter Felder der deutsch-brasilianischen Kulturbeziehungen vorgenommen wurde. Interessant ist dabei insbesondere, dass alle Themen sowohl aus einer deutschen als auch aus einer brasilianischen Perspektive behandelt werden (z. B. der brasilianische Film in Deutschland/ der deutsche Film in Brasilien), wodurch sich interessante Dialogprozesse ergeben. 
der Gemeinschaft der lusophonen Länder. Erst an dritter Stelle steht die Kulturvermittlung im Nord-Süd-Dialog, wie sie beispielsweise im Umfeld der Fußballweltmeisterschaft 2006 in Deutschland unter dem Motto Copa da Cultura stattfand.

Um die strukturelle Asymmetrie in den kulturellen Beziehungen zwischen beiden Ländern zu überwinden, haben die Teilnehmer eines deutschbrasilianischen Symposiums 2008 unter anderem vorgeschlagen, die Verbreitung des brasilianischen Portugiesisch in Deutschland durch Lektoren an deutschen Universitäten mit Förderung durch die brasilianische Regierung auszubauen, die Übersetzung von hervorragenden wissenschaftlichen und literarischen Werken mit Hilfe spezieller Programme zu fördern und durch gegenseitige Besuche und Gastaufenthalte einen systematischen Gedankenaustausch zwischen den Kulturvermittlern anzukurbeln (Bader 2010: 341 f.).

\section{Die Entwicklungspolitische Zusammenarbeit}

Deutschland ist seit einem halben Jahrhundert in Brasilien entwicklungspolitisch aktiv. Inzwischen passt das Land aufgrund seines Entwicklungsstandes und seiner gewachsenen globalen und regionalen Bedeutung immer weniger ins klassische Profil der deutschen Entwicklungszusammenarbeit (EZ). Brasilien selbst ist in den vergangenen Jahren vor allem in Lateinamerika und in Afrika zu einem wichtigen entwicklungspolitischen Geber geworden. 2006 wurde die bilaterale EZ neu ausgerichtet, um diesen Faktoren Rechnung zu tragen. Sie ist auch Teil des 2008 unterzeichneten Aktionsplans der strategischen Partnerschaft. Wichtigste Ziele der Zusammenarbeit sind die Bekämpfung des Klimawandels und der Schutz der Biodiversität, die Schwerpunkte liegen im Bereich des Schutzes des Tropenwaldes sowie der Förderung von erneuerbaren Energien und von Energieeffizienz. Auch wenn die politische Bedeutung der EZ mit Brasilien mittlerweile aus deutscher Perspektive weitaus größer ist als die finanzielle, war Deutschland 2010 der zweitgrößte bilaterale Geber des Landes. Inzwischen beteiligt sich die brasilianische Regierung allerdings je nach Thema und Region mit 30-90\% an den Kosten der EZ. Insofern weicht die EZ mit Brasilien - ohne entwicklungspolitische Ziele aufzugeben - erheblich vom Kooperationsstandard mit klassischen Entwicklungsländern ab. Sie ist nicht zuletzt geprägt durch eine forcierte Zusammenarbeit staatlicher Instanzen mit der Wirtschaft (private public 
partnerships) sowie durch gemeinsame deutsch-brasilianische EZ in Drittländern (Dreieckskooperation). ${ }^{2}$

Weitere wichtige Akteure der deutschen EZ neben den entwicklungspolitischen Durchführungsorganisationen ${ }^{3}$ sind die politischen Stiftungen. Seit 1969 ist die Konrad-Adenauer-Stiftung (KAS) in Brasilien vertreten. Ihre Aktivitäten richteten sich zunächst auf soziale Entwicklung und Demokratieförderung, seit dem Ende der Militärdiktatur 1985 nehmen das Zusammenspiel demokratischer Institutionen, die internationale Rolle Brasiliens und Fragen der nachhaltigen Entwicklung stärkeren Raum ein. Gegenwärtig konzentriert sich die Arbeit auf die Schwerpunkte internationale Politik, politische Bildung, soziale Marktwirtschaft sowie Umwelt, Klima und Energie (<www.kas. de/brasilien>). Die Friedrich-Ebert-Stiftung (FES) ist seit 1977 in Brasilien vertreten. Wichtigste Partnerorganisationen sind die Arbeiterpartei PT (Partido dos Trabalhadores) und der Gewerkschaftsdachverband CUT (Central Única dos Trabalhadores), die Projektarbeit konzentriert sich auf die Themen internationale Politik, Staat und Gesellschaft, Arbeitsbeziehungen, Gewerkschaften und soziale Inklusion (<www.fes.org.br>). Die Heinrich-Böll-Stiftung (HBS) ist seit 1990 mit Projekten in Brasilien aktiv. Sie arbeitet mit diversen NGOs, mit sozialen Bewegungen, Universitäten und Gewerkschaften zusammen. Thre Programmarbeit widmet sich schwerpunktmäßig den Achsen Menschenrechte und nachhaltige Entwicklung (<www.br.boell.org >). Die Friedrich-Naumann-Stiftung (FNS) unterhält seit 1992 ein Projektbüro in São Paulo. Im Mittelpunkt ihrer Aktivitäten steht die Zusammenarbeit mit den brasilianischen Liberalen. Die zentralen Themen der Projektarbeit 20122015 lauten Freiheit und Fortschritt, Freiheit und Religion sowie Freiheit und Partizipation (<www.ffn-brasil.org.br>). Seit 2003 ist auch die Rosa-Luxemburg-Stiftung (RLS) in Brasilien vertreten. Das Regionalbüro in São Paulo koordiniert die Aktivitäten der Stiftung in Brasilien und im Cono Suk es fördert zivilgesellschaftliche Organisationen und koordiniert eigene Veranstaltungen, Workshops, Publikationen und Ausstellungen. Zentrales Ziel der Arbeit ist die Unterstützung politischer Akteure, die sich für demokratische Partizipation und soziale Gerechtigkeit einsetzen (<www.rls.org.br>).

2 Siehe zu diesem Thema die Informationen auf der Website des Bundesministeriums für Wirtschaftliche Zusammenarbeit und Entwicklung <www.bmz.de/de/ was_wir_machen/laender_regionen/lateinamerika/brasilien/zusammenarbeit.html> (5.7.2013) sowie Stamm 2006 und Scholl/Krammenschneider 2010.

3 Zum Brasilienengagement der Deutschen Gesellschaft für internationale Zusammenarbeit (GIZ) siehe <http://liportal.giz.de/brasilien.html> (5.7.2013). 


\section{Die zivilgesellschaftlichen Beziehungen}

Deutschland und Brasilien verbindet ein breites Geflecht zivilgesellschaftlicher Beziehungen. Dazu gehören die Aktivitäten der DeutschBrasilianischen Gesellschaft, der kirchlichen Hilfswerke sowie der zahlreichen Solidaritätsgruppen, die sich mit Brasilien auseinandersetzen und in Deutschland über Brasilien informieren. Die seit 1960 bestehende Deutsch-Brasilianische Gesellschaft (DBG) ist eine private, gemeinnützige und überparteiliche Einrichtung. Sie führt Kultur- und Informationsveranstaltungen sowie Sprachkurse durch und gibt seit 1995 mehrmals jährlich die Zeitschrift Tópicos heraus, die mit aktuellen Beiträgen zu politischen, kulturellen, wirtschaftlichen, entwicklungspolitischen und ökologischen Themen ein breites Spektrum von Brasilieninteressierten in Deutschland ansprechen will (<www.topicos.net>).

Die lateinamerikanische "Theologie der Befreiung" und nicht zuletzt brasilianische Theologen wie Dom Hélder Pessoa Câmara, Leonardo Boff und Carlos Alberto Libânio Christo (Frei Betto) stießen seit Ende der 1960er Jahre auch in Deutschland auf großes Interesse. Daraus entwickelten sich im Laufe der Zeit aktive Beziehungen zwischen vielen kirchlichen Gemeinden in Deutschland und Brasilien. Die kirchlichen Hilfswerke sind mit Entwicklungsprojekten in Brasilien aktiv. Das "Bischöfliche Hilfswerk Misereor" der katholischen Kirche unterstützt in Recife, Caruaru und weiteren brasilianischen Großstädten Programme mit Straßenkindern. Andere Projekte dienen der Verbreitung klimagerechter Landwirtschaftsmethoden und der Unterstützung von brasilianischen Organisationen wie der "Bischöflichen Kommission für Landpastoral" (CPT) oder der "Bewegung der landlosen Bauern" (MST). Die evangelische Aktion "Brot für die Welt" fördert in Brasilien vor allem Programme in den Bereichen Landwirtschaft, Ernährung und Friedenssicherung. Neben der Projektarbeit ist die entwicklungspolitische Lobbyarbeit in Deutschland und Brasilien ein Schwerpunkt der Aktivitäten der kirchlichen Organisationen, die sich in diesem Zusammenhang auch immer wieder mit Forderungen an die Regierungen der beiden Länder gewandt haben. Auch Solidaritätsaktionen mit brasilianischen Umwelt- und Menschenrechtsaktivisten sind durch die kirchlichen Hilfswerke unterstützt worden.

Seit den 1970er Jahren entwickelte sich in Deutschland eine breite zivilgesellschaftliche Solidaritätsarbeit mit Brasilien. Bis zum Ende der Militärherrschaft galten die Aktivitäten der entsprechenden Gruppen vor allem 
dem Kampf für eine Demokratisierung des Landes. Nach der Rückkehr zur Demokratie richtete sich das Augenmerk dann verstärkt dem Kampf gegen soziale Ungleichheit und Ungerechtigkeit. Zur Solidaritätsbewegung gehören sehr unterschiedliche Organisationen, beispielsweise kirchliche Gruppierungen, gemeinnützige Vereine, Netzwerke, Arbeitsgruppen aus Schulen und Universitäten, gewerkschaftliche Arbeitskreise, politische Verbände und entwicklungspolitische Organisationen. Diese Gruppen setzen sich in Deutschland für brasilianische Basisbewegungen und für die Demokratisierung und nachhaltige Verbesserung der sozialen, ökonomischen und ökologischen Lebensbedingungen in Brasilien ein. Etwa 40 Gruppen und eine gleich große Zahl von Einzelpersonen schlossen sich 1989 zum Netzwerk "Kooperation Brasilien" (KoBra) zusammen. Die Mitglieder von KoBra leisten Öffentlichkeitsarbeit, bemühen sich um politischen Einfluss und unterstützen Projekte in Brasilien. Sie arbeiten mit einer Vielzahl sozialer Bewegungen in Brasilien zusammen. KoBra versteht sich als Sprachrohr und Lobbyinstanz der Brasiliengruppen in Deutschland. Das Netzwerk fördert und unterstützt die Anerkennung und Wahrung der politischen, wirtschaftlichen, sozialen und kulturellen Menschenrechte in Brasilien, insbesondere benachteiligter Gruppen, die Entwicklung gerechter Beziehungen zwischen den Geschlechtern sowie den Kulturaustausch und die interkulturelle Arbeit. KoBra publiziert in unregelmäßigen Abständen thematische Reader und bringt zehnmal jährlich die Zeitschrift Brasilicum mit aktuellen Nachrichten aus Brasilien zu Politik, Wirtschaft und Umwelt heraus (<www.kooperation-brasilien.org $>$ ).

Die Zivilgesellschaften Deutschlands und Brasiliens sind vielfältig und alles andere als homogen. Auch die bilateralen Beziehungen zwischen den entsprechenden Akteuren in beiden Ländern sind sehr vielschichtig. Punktuell kommt es zwischen den verschiedenen Akteuren auf deutscher Seite - dem DBG, den politischen Stiftungen, kirchlichen Hilfswerken und Solidaritätsgruppen - immer wieder zu gemeinsamen Aktionen. Es gibt allerdings auch Konflikte und Widersprüche, die unter anderem auf unterschiedlichen politischen und ideologischen Vorstellungen und den daraus resultierenden Einschätzungen der Entwicklungen in Brasilien basieren. Diese pluralistische Vielfalt verbietet es einzelnen Akteuren, einen Monopolanspruch auf die Repräsentation der Zivilgesellschaft oder des zivilgesellschaftlichen Dialogs zwischen beiden Ländern zu erheben. Gerade dies macht aber auch den Reichtum der zivilgesellschaftlichen Beziehungen zwischen Deutschland und Brasilien aus. 


\section{Fazit}

Wenn in den deutschen Medien die Rede von Brasilien ist, stehen oft die Themen Fußball, Samba, Karneval, Traumstrände und tropischer Regenwald im Vordergrund. All dies gehört zu Brasilien und es ist nicht weiter verwunderlich, dass für zahlreiche Deutsche, die Brasilien vor allem als Urlaubsland betrachten, derartige Aspekte im Vordergrund stehen. Die deutsch-brasilianischen Beziehungen jedoch sind weit mehr als das. Sie zeichnen sich durch eine große Dichte und Vielfalt sowie durch die Beteiligung zahlreicher staatlicher und nichtstaatlicher Akteure aus. In vielen Bereichen bestehen weiterhin historisch überkommene Asymmetrien, aber es zeichnen sich auch Tendenzen ab, die für die Zukunft zumindest teilweise eine stärkere Gleichgerichtetheit der wechselseitigen Austauschund Transferprozesse erwarten lassen.

\section{Literaturverzeichnis}

AKTIONSPLAN DER DEUTSCH-BRASILLANISCHEN STRATEGISCHEN PARTNERSCHAFT (2008). Online zugänglich unter <www.brasil.diplo.de/Vertretung/brasilien/de/07_Aussenpolitik/ Bilaterale_Vertraege/Bilaterale_Vertraege.html> (3.7.2013).

Althoff, Gabriele (2010): "Wissenschaftliche Kooperation und akademischer Austausch". In: Costa et al., 684-699.

Auswärtiges Амт (2010): Deutschland, Lateinamerika und die Karibik: Konzept der Bundesregierung. Berlin: Auswärtiges Amt.

- (2012): Globalisierung gestalten - Partnerschaften ausbauen - Verantwortung teilen. Konzept der Bundesregierung. Berlin: Auswärtiges Amt.

Alzamora, Geane (Hg.) (2008): Kulturdialoge Brasilien - Deutschland: Design, Film, Literatur, Medien. Berlin: Ed. Tranvía.

BADER, Wolfgang (Hg.) (2010): Deutsch-brasilianische Kulturbeziebungen: Bestandsaufnahme, Herausforderungen, Perspektiven. Frankfurt am Main: Vervuert.

BoeckH, Andreas (2006): 'Der 'aufstrebende Gigant' und die Bundesrepublik Deutschland: Zum Stellenwert der deutsch-brasilianischen Beziehungen”. In: Martius-Staden-Jahrbuch, 53, 239-260.

BoeckH, Andreas/Sevilla, Rafael (Hg.) (1997): Bestandsaufnabme und Perspektiven der deutschbrasilianischen Beziehungen. Frankfurt am Main: TFM.

BRANDÃo OsORIO, Luiz Felipe (2011): “O sentido estratégico das relações bilaterais BrasilAlemanha”. Dissertação de Mestrado apresentada ao Programa de Pós-Graduação em Economia Política Internacional. Universidade Federal do Rio de Janeiro. 
Bundesverband der Deutschen Industrie (2012): BDI Brazil Board. Ziele. Themen. Mitglieder. Berlin: BDI.

Costa, Sérgio/Kohlhepp, Gerd/Nitschack, Horst/Sangmeister, Hartmut (Hg.) (2010): Brasilien heute. Geographischer Raum · Politik · Wirtschaft · Kultur. 2. vollständig neu bearbeitete Auflage. Frankfurt am Main: Vervuert.

DantAS, Marcelo (2010): "Kultur und auswärtige Kulturpolitik in Brasilien”. In: Bader, 333-337.

Dungen, Johannes von (2010): Vom Freund zum Partner. Die deutsch-brasilianischen Kulturbeziebungen im Wandel. Stuttgart: ifa.

Gate Germany (2009): Länderprofile. Analysen - Erfahrungen - Trends. Edition Brasilien. Bonn: DAAD.

Germany Trade \& Invest (2013): Wirtschaftsdaten kompakt: Brasilien. Bonn. <www.gtai. de> (3.7.2013).

GÖBEL, Barbara/BiRLE, Peter/SPECHT, Johannes (2009): Wirtschafts-, sozial- und geisteswissenschaftliche Lateinamerikaforschung in Deutschland. Situation und Perspektiven. Berlin: IberoAmerikanisches Institut.

Pinheiro Guimarães, Samuel (Hg.) (2000): Alemanha: visões brasileiras. Brasília: Instituto de Pesquisa de Relações Internacionais/Fundação Alexandre de Gusmão.

Lohbauer, Christian (2000): Brasil - Alemanha: 1964 - 1999. Fases de uma parceria. São Paulo: Konrad-Adenauer-Stiftung.

Menezes, Albene/Kотнe, Mercedes (1997): Brasil e Alemanha: 1827 - 1997: Perspectivas Históricas. Brasília: Thesaurus.

Moniz Bandeira, Luiz Alberto (1995): Das deutsche Wirtschaftswunder und die Entwicklung Brasiliens: die Beziehungen Deutschlands zu Brasilien und Lateinamerika (1949 - 1994). Übersetzt von Marie-Louise Sangmeister-Plehn. Frankfurt am Main: Vervuert.

Moniz Bandeira, Luiz Alberto/Pinheiro Guimarães, Samuel (Hg.) (1995): Brasil e Alemanha: a construção do futuro. Brasília: Instituto de Pesquisa em Relações Internacionais, Fundação Alexandre de Gusmão.

RÖDl \& PARTNer (2012): Perspektiven verbessern. Going Global. Der deutsche Mittelstand in Brasilien 2012. Nürnberg.

SANGMEISTER, Hartmut/SCHÖNSTEDT, Alexa (2010): "Die brasilianisch-deutschen Wirtschaftsbeziehungen. Aus Tradition in die Zukunft: Solide Geschäfte zwischen ungleichen Partnern". In: Costa et al., 656-667.

SCHOLL, Johannes/KRAMMENSCHNEIDER, Ulrich (2010): "Deutsche Entwicklungszusammenarbeit mit Brasilien”. In: Costa et al., 669-682.

StAmm, Andreas (2006): Entwicklungszusammenarbeit im Gesamtkontext der Deutsch-Brasilianischen Kooperation. Eine Portfolioanalyse. Discussion Paper 19. Bonn: Deutsches Institut für Entwicklungspolitik.

VARGAS, Everton Vieira (2011): "Brasil-Alemanha: uma parceria para ousar". In: Valor Econômico (19. September).

VAZ, Alcides Costa (1999): "Parcerias estratégicas no contexto da política exterior brasileira: implicações para o Mercosul.” In: Revista Brasileira de Política Internacional, 42, 2, 52-80. 